\title{
UAV Remote Sensing for delineation of Urban Vegetation using Object Based Image Analysis
}

\author{
Munasinghe N., Manatunga U.I*., Premasiri H.M.R. \\ Department of Earth Resources Engineering, University of Moratuwa, Sri Lanka \\ *mana.udeshini919@gmail.com
}

\begin{abstract}
Remote sensing technology has rapidly advanced during the last few decades and the number of remote sensing platforms has increased. The development of Unmanned Aerial Vehicles (UAV) image acquisition systems has radically changed the aerial Photogrammetric mapping due to its low cost, high spatial resolution and high accuracy and provide a great potential for vegetation mapping in urban areas. Urban environment planning becomes a challenging task for urban planners due to fast urbanization processes and growth of population. Urban land use is a crucial information for planning authorities and there is a growing demand for urban land cover maps for decision-making procedures in urban planning. In this article, we demonstrate a rule sets knowledge-based classification method, in object oriented classification which is a fully automated and highly accurate engineering approach for demarcation of urban vegetation with the use of eCognition software. DJI Phantom 4, consumer grade drone was used to acquire high resolution aerial photos as an input dataset in the study. In this study, vegetation mapping was done using the textural and contextual information acquired from the RGB image (Orthophoto) without using any Near Infrared (NIR) information and a Digital Surface Model (DSM) which was developed using Pix4D software, used as an ancillary data in the classification process in order to obtain the elevation information. The extraction of tree canopy and the buildings in a coastal urban environment has used to illustrate the analysis. The DSM was validated using ground control points observed by field measurements. The resultant urban vegetation map was validated with the digitized land use layer and an overall accuracy of $90.15 \%$ was obtained. This study indicates that low cost drones compared to the survey grade drones, also can provide high accurate and high resolution products suitable for many urban local area planning studies.
\end{abstract}

Keywords: Photogrammetry, Object oriented classification, DSM, Orthophoto, Urban planning 